\title{
Statistically bias-corrected and downscaled climate models underestimate the severity of U.S. maize yield shocks
}

\section{David Lafferty ( $\square$ davidcl2@illinois.edu )}

University of Illinois at Urbana-Champaign https://orcid.org/0000-0003-2098-6885

\section{Ryan Sriver}

University of Illinois https://orcid.org/0000-0001-9587-3741

Iman Haqiqi

Purdue University https://orcid.org/0000-0001-7331-4615

\section{Thomas Hertel}

Purdue University

\section{Klaus Keller}

The Pennsylvania State University

\section{Robert Nicholas}

The Pennsylvania State University https://orcid.org/0000-0003-2615-2574

\section{Article}

Keywords: climate change, agriculture, climate models

Posted Date: February 19th, 2021

DOI: https://doi.org/10.21203/rs.3.rs-157774/v1

License: (1) This work is licensed under a Creative Commons Attribution 4.0 International License. Read Full License

Version of Record: A version of this preprint was published at Communications Earth \& Environment on September 20th, 2021. See the published version at https://doi.org/10.1038/s43247-021-00266-9. 


\section{Statistically bias-corrected and downscaled climate models underestimate the severity of U.S. maize yield shocks}

\section{Abstract}

2 Efforts to understand and quantify how a changing climate can impact agriculture often

3 rely on bias-corrected and downscaled climate information, making it important to quantify

4 potential biases of this approach. Previous studies typically focus their uncertainty

5 analyses on climatic variables and are silent on how these uncertainties propagate into

6 human systems through their subsequent incorporation into econometric models. Here,

7 we use a multi-model ensemble of statistically downscaled and bias-corrected climate

8 models, as well as the corresponding CMIP5 parent models, to analyze uncertainty

9 surrounding annual maize yield variability in the United States. We find that the CMIP5

10 models considerably overestimate historical yield variability while the bias-corrected and

11 downscaled versions underestimate the largest historically observed yield shocks. We

12 also find large differences in projected yields and other decision-relevant metrics

13 throughout this century, leaving stakeholders with modeling choices that require

14 navigating trade-offs in resolution, historical accuracy, and projection confidence. 


\section{Introduction}

19 Understanding and managing climate risk hinges on a quantitative description of the

20 human and Earth system dynamics as well as their associated uncertainties ${ }^{1}$. The fidelity

21 and utility of such analyses depend on using appropriate underlying climate information.

22 While global climate models (GCMs) can provide useful insights at the global scale, they

23 can face severe problems at regional to local scales. There are two main reasons for this.

24 For one, the native resolution of GCMs is too coarse to be used in fine-scale analysis ${ }^{2}$. In

25 addition, GCMs exhibit systematic biases relative to observations that require projections

26 to be appropriately corrected and interpreted ${ }^{3,4}$.

As a result, bias-corrected and downscaled climate products are widely employed

28 for a broad range of end-use applications, including diagnosing the impacts of climate

29 change on the economy ${ }^{5}$, energy demand ${ }^{6}$, and human populations ${ }^{7}$; accounting for

30 climate change in regional infrastructure planning 8,9 ; and devising climate change

31 adaptation strategies from the national level ${ }^{10}$ to city and urban areas ${ }^{11}$.

Although generally regarded as 'value-added' with respect to raw GCM outputs ${ }^{12}$,

33 bias-corrected and downscaled climate products still contain considerable uncertainties.

34 Examples include the validity of the stationarity assumption underpinning many bias-

35 correction and downscaling methods ${ }^{13,14}$, the physical plausibility of results if methods are

36 applied without expert knowledge ${ }^{15}$, the resulting representation of atmospheric or

37 hydrologic variability ${ }^{16,17}$, and the applicability of methods in a changing climate ${ }^{18,19}$. It is

38 important to understand how these uncertainties might propagate through econometric

39 models and influence estimates of socioeconomic outcomes. Many previous studies in the

40 climate and hydrology literatures focus on developing new bias-correction and

41 downscaling techniques ${ }^{20-22}$ or evaluating the efficacy of existing ones ${ }^{23-25}$. However, few

42 perform an 'end-to-end' analysis that incorporates sector-specific models of climate 43 impacts $^{26}$.

44 Here, we analyze how uncertainties from one statistical bias-correction and 45 downscaling technique propagate into simulated agricultural outcomes by employing a 46 parsimonious regression model of maize yields adapted from ref. ${ }^{27}$. In keeping with a 47 substantial literature documenting the highly nonlinear effects of temperature on maize 
48 yields ${ }^{27-30}$, we account for the influence of temperature via growing degree days (GDDs),

49 which are beneficial for yields, and extreme degree days (EDDs), which are indicative of 50 heat stress and can severely reduce yields. Further relying on previous works ${ }^{31}$, we also

51 include a quadratic term in season-total precipitation. This model can reproduce yield 52 records from the United States Department of Agriculture (USDA) with good accuracy (see

53 Materials and Methods). We run the yield model across the continental United States

54 (CONUS) using a multi-model ensemble of bias-corrected and downscaled climate 55 models from the NASA Earth Exchange Global Daily Downscaled Projections (NEX56 GDDP) datase ${ }^{32}$, as well as with the corresponding parent GCMs from the Coupled Model 57 Intercomparison Project Phase 5, or CMIP5 $5^{33}$. By comparing yield hindcasts over the 58 historical period (1960-2005) to yields simulated by an observational dataset, we quantify 59 uncertainty due to bias-correction and downscaling as it pertains to crop yield outcomes.

60 Using a high-emissions scenario, we then project yields using both ensembles throughout 61 the remainder of this century and discuss notable differences and their implications. Our 62 analysis highlights the importance of uncertainty propagation from global models through 63 bias-correction and downscaling to its effect on simulated yield variability and other 64 decision-relevant metrics.

\section{Results and Discussions}

Hindcast Evaluation of the National Yield Distribution. Simulations based on the original CMIP models considerably overestimate the variability of the observed CONUS yield distribution. Conversely, using the bias-corrected and downscaled ensemble (NEXGDDP) underestimates the severity of the largest historically observed yield shocks.

72 Figure 1.a shows the modeled national-level probability distribution of yield anomalies for 73 each multi-model ensemble, constructed by aggregating county-level yields using yearly 74 production weights. To facilitate a clearer diagnosis of the associated uncertainties, we 75 use yields simulated by the observational climate product as 'observed yields', rather than 76 actual USDA records. Further, we choose the same observational climate dataset that 77 was used to bias-correct and downscale the original models. Figure 1.a shows that while 78 the NEX-GDDP ensemble exhibits excellent agreement with the observed histogram over 79 the central portion of the distribution, the CMIP ensemble gives a better representation of 
80 the left or lower yield tail-particularly for the two most extreme shocks over the historical 81 period.

82 From the probability distributions in Figure 1.a, we also compute sampling 83 distributions of selected summary statistics and compare them with the observed values,

84 shown in Figures 1.b-d. To test how well each ensemble captures the observed variability, 85 with a particular emphasis on the tails of the distribution, we choose as summary statistics 86 the standard deviation (Figure 1.b), median absolute deviation (Figure 1.c), and magnitude 87 of the largest negative yield shock (Figure 1.d). To translate into a quantitative measure, 88 the tail-area probability $\left(p_{\text {tail }}\right)$ is calculated as the right-tail probability for each distribution, 89 measured from the observed value. Figure 1.b shows that in only $17 \%$ of cases does the 90 NEX-GDDP ensemble generate a sample with a standard deviation at least as large as 91 the observed value. This rises to $68 \%$ for the median absolute deviation (Figure 1.c), a 92 more robust measure of variability that is insensitive to outliers, which here are the largest 93 yield shocks. By contrast, every sample generated by the CMIP ensemble exhibits 94 variability, measured either by the standard deviation or median absolute deviation, larger 95 than that observed.

96 Decision-making strategies that are robust against low-probability, high-impact 97 events require information about the entire range of outcomes ${ }^{34}$. Here, the largest 98 negative yield shock (Figure 1.d) represents the most extreme event observed throughout

99 the historical period. Consistent with the CMIP-based hindcasts overestimating the 100 variability of the historical distribution, they also overestimate the severity of the largest 101 shock, with $97 \%$ of samples exhibiting a greater magnitude than that observed. 102 Conversely, the tendency of the NEX-GDDP ensemble to underestimate variability is 103 exacerbated here-in only $5.7 \%$ of samples is the simulated shock as large as observed.

104 Thus, while it is clear that bias-correction and downscaling lead to an overall improvement 105 in the representation of national yield variability, this improvement is concentrated in the 106 central portion of the distribution. For the tails, the net effect is an over-correction of the 107 original large variability bias, resulting in an under-sampling of the most extreme weather108 induced shocks.

110 Hindcast Evaluation at the County Level. To what extent are the national-level results 111 replicated across individual counties in CONUS? We find that for many counties, 112 particularly in and around the 'Corn Belt' region encompassing historically high-yield 113 counties (Figure S1), conclusions from the national level hold. Figure 2 shows a map of 
114 yield tail-area probabilities calculated from NEX-GDDP for each of the summary statistics

115 in Figure 1. Small tail-area probabilities for the standard deviation (Figure 2.a) 116 demonstrate that driving our yield model with the bias-corrected and downscaled 117 ensemble underestimates the bulk variability of observed yields for many important maize-

118 growing counties. As indicated by the 1\% stippling, NEX-GDDP grossly underestimates

119 the variability for several counties in Illinois and lowa which are among the highest-

120 production counties in CONUS (Figure S1). We observe that the median absolute

121 deviation is underestimated for fewer counties (Figure 2.b) but the magnitude of the largest

122 negative shock is underestimated for more counties, again indicating that the central part

123 of the observed distribution is better captured than the tails.

124 The equivalent maps for the CMIP ensemble (Figure S2) reveal that the parent 125 models systematically overestimate historical yield variability, displaying large tail-area 126 probabilities for all summary statistics across a majority of maize-growing counties. Thus, 127 we again conclude that bias-correction and downscaling lead to an under-sampling of the 128 tails of the yield distribution. Further, we find that this holds across a large number of high129 yield counties in CONUS, which is why this bias persists at the national level.

131 Hindcast Evaluation of Climate Variables. What is the source of this low-variability bias 132 in the NEX-GDDP yield hindcasts? Recall that for yield observations, we use the results 133 of driving the statistical yield model with the same observational product that was used in 134 the bias-correction and downscaling. The yield model itself can hence be eliminated as a 135 source of uncertainty. Instead, this bias is driven by the representation of each of the 136 underlying climate variables in the model. Specifically, we find that the NEX-GDDP 137 ensemble underestimates the variability of extreme degree days. It performs better with 138 the more moderate temperature variable and the precipitation variable. Figure 3 shows 139 the map of tail-area probabilities calculated from the NEX-GDDP ensemble for each 140 climate variable across the eastern half of CONUS, with the standard deviation and 141 median absolute deviation as summary statistics. Both temperature-derived variables 142 show a predominantly latitudinal gradient in tail-area probabilities with variability being 143 underestimated farther north and overestimated to the south. The NEX-GDDP ensemble 144 shows a strong prevalence toward underestimating the variability of both variables 145 throughout the Corn Belt region, which is the primary driver of the resulting low-variability 146 bias in the corresponding maize yield hindcasts. However, as indicated by the $1 \%$ 
147 stippling, the representation of extreme degree days, defined as an integrated measure of

148 temperature above $29^{\circ} \mathrm{C}$, is considerably worse.

149 For season-total precipitation, there is less evidence of a clear bias that would 150 affect maize yields-the most prominent feature is a tendency to overestimate the 151 standard deviation across many central and southern counties. One potentially fruitful 152 avenue for future work is to investigate the effects of bias-correction and downscaling on 153 the representation of soil moisture, which is more important for plant growth ${ }^{35}$ and may or 154 may not be strongly correlated with cumulative precipitation depending on factors such as 155 runoff, drainage, and irrigation ${ }^{36}$.

156 All tail-area probabilities generally cluster toward more moderate values when the 157 median absolute deviation is considered, as demonstrated by the reduction in $1 \%$ 158 stippling. Since the median absolute deviation is insensitive to outliers, this behavior is 159 consistent with the hypothesis that the NEX-GDDP ensemble tends to perform better at 160 capturing centrally located observations, now for each climate variable in addition to for 161 the yield distribution itself. However, for many of the most northern counties, NEX-GDDP 162 underestimates even the median absolute deviation for the extreme temperature variable. 163 As might be expected, the original CMIP models overestimate historical variability 164 for each climate variable for almost every county in the eastern half of CONUS (Figure 165 S3). The effects of bias-correction and downscaling hence show a clear spatial pattern. 166 For extreme degree days, the large variability bias is over-corrected for the most northern 167 counties with the coldest climates and the smallest historical variability (Figure S4). In 168 contrast, the large-variability bias is under-corrected for many southern counties with 169 warmer climates and larger historical variability, while the best performance is observed 170 for central counties. These patterns are also broadly exhibited for the more moderate 171 growing degree days. Thus, as a clear example of uncertainty propagation, the low 172 variability bias in NEX-GDDP simulated yields results from a tendency of this statistical 173 bias-correction and downscaling technique, for many important maize-growing counties in 174 CONUS, to over-correct the large-variability bias inherent to CMIP. We further highlight 175 that an analysis based on temperature itself would not uncover this NEX-GDDP bias, 176 which arises only for the degree day variables used in the yield model.

178 Yield Projections. Decision-makers care about the reliability of future climate projections, 179 for which adequate past performance is generally regarded as a necessary but insufficient 
condition $^{37}$. One hypothesis, based on the tendency of the NEX-GDDP ensemble to underestimate historical yield variability, is that projections are similarly overconfident.

There are considerable differences between the yield projections of the NEX-

183 GDDP and CMIP ensembles. Figure 4.a shows the projected national-level distributions

184 for each multi-model ensemble for two 20-year periods during the 21st century: 20401852059 (mid-century) and 2080-2099 (late century). We use the relatively high-emissions

186 Representative Concentration Pathway (RCP) 8.5 scenario $^{38}$ to emphasize the 187 differences between the ensembles. One robust response is that all four projections show 188 an increase in yield variability relative to historical observations (shown in gray). However, 189 as with the historical hindcasts, the NEX-GDDP ensemble exhibits reduced variability 190 relative to the original CMIP models. Although these projection differences alone do not demonstrate conclusively that the bias-corrected and downscaled models are

193 they do illustrate the importance of end-users' modeling choices, particularly in light of the 194 results discussed in previous sections.

195 To illustrate how projection differences might affect decision-making, we consider 196 how the different distributions affect estimates of return periods as a function of yield shock 197 magnitude. Return periods give the average time between (often rare and damaging) 198 events and are used often as part of risk analysis ${ }^{40}$. In our case, we estimate the average number of years between large national-level yield drops driven by weather and climate. Figure 4.b shows that the calculated return periods from the CMIP and NEX-GDDP ensembles diverge for increasingly large yield shocks, particularly around the middle of the century. As an example, consider the magnitude of the largest national-level shock throughout the 46-year historical period, around 0.3. NEX-GDDP projects that an event this size will occur approximately every 16 years by the middle of the century; CMIP projects roughly every 7 years. By the end of the century, NEX-GDDP projects a shock this size approximately once every 7 years and for CMIP, roughly every 5 years. This presents a challenge for decision-makers in deciding which product to rely on, given that low-probability, tail events can have disproportionate negative impact. For example, it might pay to hold commodity stocks under NEX-GDDP but not CMIP. Alternatively,

210 investing in adaptation measures might pass a cost-benefit analysis under CMIP, but not 211 NEX-GDDP.

\section{Caveats and Conclusions}


215 Climate models are increasingly used to support regional impact analysis. 216 Understanding, quantifying, and ultimately reducing the uncertainties and biases in 217 climate impact projections is therefore crucial to improving decision-making. Bias218 corrected and downscaled GCM output represents the state-of-the-art of climate modeling 219 and many such datasets are used widely in the scientific literature as well as by decision220 makers in public and private sectors. It is important to understand how uncertainty 221 associated with methods of bias-correction and downscaling might propagate from the 222 underlying climate information through models of human systems and influence key 223 metrics of interest to stakeholders.

224 We analyze the effects of bias-correction and downscaling on modeled maize 225 yields throughout the continental United States. Using a transparent, fast, and skillful 226 statistical yield model and minimizing the influence of other factors, we find that the NEX227 GDDP ensemble underestimates the largest historically observed negative yield shocks. 228 We can attribute this effect to the process of bias-correction and downscaling of the 229 original GCM outputs. By further evaluating the representation of each climate variable in 230 the yield model, we also provide qualitative evidence that this underestimation of variability 231 can be traced to temperature extremes in the form of extreme degree days. This 232 uncertainty also has important implications regarding the use of projections-yield 233 variability and associated risk metrics are considerably reduced in downscaled and bias234 corrected projections relative to the parent models. This presents end-users with non235 trivial decisions regarding which models to use. Choosing a climate projection dataset 236 involves navigating potentially harsh trade-offs among resolution, historical performance 237 of bulk metrics and extremes, and possible overconfidence.

238 Our study has important caveats that point to future research needs. For one, we 239 consider projections based on a relatively simple statistical downscaling and bias240 correction method. Our conclusions are hence of limited relevance to potentially more 241 skillful bias-correction and downscaling techniques. Relative to the NEX-GDDP method, 242 dynamic downscaling approaches are better suited for regions of complex terrain ${ }^{41}$ and 243 more sophisticated statistical approaches may give a better representation of atmospheric 244 variability ${ }^{41,42}$. Second, our conclusions may not be valid for all econometric models that 245 employ bias-corrected and downscaled climate information. It may be possible that 246 structurally different yield models from the one employed here-for example, using 247 average seasonal temperature rather than degree days-are less susceptible to biases in 
248 the NEX-GDDP ensemble. Beyond the agricultural sector, econometric models will, in 249 general, be sufficiently different as to merit a separate investigation into whether biases

250 persist that are similar to those uncovered here. Given the widespread and 251 disproportionate impacts of extreme heat on human systems ${ }^{43}$, these investigations would 252 be worthwhile. Third, we have treated each ensemble as a 'model democracy' where each 253 model output is weighted equally. There is a growing literature on more sophisticated 254 weighting schemes that account for model dependence and performance such that key 255 ensemble metrics are optimized ${ }^{44}$. Such schemes could be applied here with the aim of 256 ensuring that yield hindcasts and projections are not overconfident, for example. Finally, 257 we assume no future adaptation in yield projections and do not account for increased 258 atmospheric carbon dioxide concentrations, which mitigate yield declines ${ }^{45}$. Recall that 259 Figure 4 shows climate- and weather-driven yield deviations around the technological time 260 trend, meaning that even in years with large shocks, the absolute yield could still be higher 261 than today's levels. Figure 4 should not be taken as a prediction but rather as a 262 demonstration of the differences that arise from bias-correction and downscaling when 263 subsequently applied to a historically realistic yield model.

264 Given these caveats, this work raises important questions about the use of bias265 corrected and downscaled climate information. Without careful consideration of the 266 representation of low-probability, high-impact events, the use of bias-corrected and 267 downscaled climate projections may lead to underestimates of the severity of impacts and 268 consequently, poor decisions. This is especially true for coupled human-environment 269 systems where extremes may be sensitive to feedbacks. In the agricultural sector, for 270 example, land-use change or irrigation practices can affect the local hydroclimatology ${ }^{46,47}$.

271 Finally, this work highlights the importance of taking a holistic approach and using metrics

272 specific to the sector of interest when evaluating the efficacy of bias-corrected and 273 downscaled climate products.

\section{Methods}

277 Climate Data. The NEX-GDDP dataset consists of statistically bias-corrected and 278 downscaled climate scenarios derived from 21 different CMIP5 models. One simulation 279 member from each CMIP5 model was used to derive historical hindcasts (1950-2005) and 280 future projections (2006-2100) of daily maximum and minimum temperature and mean 281 precipitation, with projections run under RCPs 4.5 and 8.5. From the varying spatial 
282 resolutions of the parent CMIP5 models (see Table S1), all downscaled outputs have a 283 constant lateral resolution of $0.25^{\circ}$. The observational dataset used was the Global 284 Meteorological Forcing Dataset ${ }^{48}$. The bias-correction and downscaling were performed 285 via the Bias-Correction Spatial Disaggregation algorithm ${ }^{32}$. Further details can be found in 286 the SI Text. Academic studies that employ the NEX-GDDP dataset include refs. ${ }^{6,49-52}$.

288 Yield Model Construction and Evaluation. The concept of degree days is a widely used 289 measure for the amount of cumulative heat a crop is exposed to over the length of the 290 growing season, which in this work is held constant at March through August. Degree days 291 are derived for each grid cell by sinusoidally interpolating the diurnal temperature cycle 292 using maximum and minimum daily temperatures (a detailed derivation can be found in 293 ref. ${ }^{31}$ ). GDDs are then calculated as the area under this temperature (T) curve subject to 294 the bounds $10^{\circ} \mathrm{C}<\mathrm{T} \leq 29^{\circ} \mathrm{C}$, summed over the growing season. EDDs are defined 295 similarly, but with $\mathrm{T}>29^{\circ} \mathrm{C}$. These bounds are specific to maize ${ }^{27}$. An area average is 296 used to convert from the climate model or observational grid to the county level.

297 Modeling the yield sensitivity to moisture poses greater challenges. Complex daily 298 interactions between soil moisture extremes and heat stress can lead to 299 drastically different yield outcomes ${ }^{36}$. As discussed, season-total precipitation is not 300 always strongly correlated with seasonal mean soil moisture. Precipitation fields are also 301 inherently noisier than temperature fields in climate reanalysis products, further 302 complicating the extraction of the response function. Previous works rely on cumulative 303 precipitation ${ }^{53,54}$. To keep the yield model simple and fast, we include here only a quadratic 304 season-total precipitation term.

305 Our resulting yield model is then:

$$
\log Y_{i, t}=f_{i}(t)+c_{i}+\alpha_{i} G D D_{i, t}^{\prime}+\beta_{i} E D D_{i, t}^{\prime}+\gamma_{i} P_{i, t}^{\prime}+\kappa_{i} P_{i, t}^{\prime 2}+\epsilon_{i, t} .
$$

307 Here, $i$ denotes the spatial index (for this analysis, each county in CONUS), $t$ denotes the 308 temporal index (each year), $Y$ is the yield in bushels/acre, GDD denotes growing degree 309 days, EDD denotes extreme degree days, and $P$ denotes total in-season precipitation. A 310 quadratic, county-specific time trend, $f_{i}(t)$, is included to account for technological 311 progress and a constant term, $c_{i}$, is included to account for time-invariant group fixed 312 effects such as soil quality. A prime indicates that the sample mean is removed. The 
313 residual error is given by $\epsilon$ and assumed to be Gaussian, independent and identically 314 distributed.

315 We use yearly county-level yield records to fit the coefficients in Eq. (1) using an 316 ordinary least squares regression subject to the constraints $\alpha>0, \beta<0, \gamma>0, \kappa<0$. For

317 each county, the group fixed effect $c_{i}$ is simply the mean of the de-trended yield time

318 series, so the regression need only calculate the coefficients in front of the four climate 319 variables.

320 Other modeling choices are possible here-our regression implementation is 321 straightforward, and in constraining the coefficients to be the correct sign we rely on prior 322 knowledge from previous works. Bayesian methods would allow a more sophisticated 323 approach to represent prior knowledge. Additionally, the fit residuals are correlated in 324 space (Figures S5, S6). More sophisticated treatments of the residual structure are 325 possible ${ }^{55}$, but we choose a simple model-fitting approach for parsimony.

326 Given these caveats, we fit this model using 1960-2005 as the training period and 327 2006-2016 for an out-of-sample validation, since 2005/2006 marks the hindcast/projection 328 boundary in the climate model runs. Additionally, the fitting is performed only for counties 329 that exhibit at least $50 \%$ data coverage in the USDA record over the training period. Model 330 skill is assessed with the coefficient of determination, $R^{2}$, calculated without the inclusion 331 of the time trend (i.e., it provides only a measure of how well the model captures the year332 to-year weather-induced variations). We analyze spatial maps of $R^{2}$ at the county level as 333 well as at the national level after aggregating by county production share, where 334 production is defined as yield multiplied by harvested area. Additionally, we test whether 335 Eq. (1) represents an improvement over only the quadratic time trend by using leave-one336 out cross-validation (for a more detailed description, see the SI Text). In all analyses 337 involving the modeled yields, we include only the counties where including the climate 338 variables gives a lower mean squared error. This is to ensure that our conclusions are 339 drawn from counties where including climate data in the model is meaningful. Equivalents 340 of Figures 1, 2, and 4 that include all counties are shown in Figures S7, S8, and S9, 341 respectively.

342 Our yield model is able to reproduce USDA-recorded yields with good accuracy 343 (Figure S10). In the training period, the median $R^{2}$ for all 2,371 counties is 0.35 ; for the 3441,816 counties where including the climate variables improved the fit, the median $R^{2}$ is 3450.41 (these counties made up no less than $80 \%$ of total national-level production since 346 1960-see Figure S11). The equivalent out-of-sample values are 0.14 and 0.20 , 
347 respectively (Figure S12). The best fits are consistently found in and around the Corn Belt

348 region, which is why the national-level time series of aggregated yields also exhibits good

349 agreement with USDA records. The national-level $R^{2}$ for the training period is 0.49 and for

350 the out-of-sample period is 0.71 .

352 Evaluation Metric. In evaluating the climate model hindcasts, it is important to note that 353 the time series of simulated yields are generally out of phase with observed yields on a 354 year-by-year basis. As such, we choose an evaluation metric that is insensitive to the 355 temporal dimension of modeled yields and instead focuses on long-term distributions. For 356 each multi-model ensemble, we estimate the yield probability density function for each 357 county in CONUS by constructing a kernel density estimate of the 966 (21 models x 46 358 years) point values. Then, $10^{4}$ samples of length equal to the observational data are 359 drawn and for each summary statistic, a sampling distribution is compiled. These sampling 360 distributions are displayed graphically for the national level in Figure 1. At the county level, 361 we report the tail-area probability, calculated as

$$
p_{\text {tail }}=\operatorname{Pr}\left(S\left(y^{e n s}\right) \geq S\left(y^{o b s}\right)\right) .
$$

363 Here, $S\left(y^{o b s}\right)$ is a given summary statistic of the observed distribution and the probability 364 is understood to be taken over multiple realizations of the simulated distribution for each 365 ensemble, $y^{e n s}$. Thus, the tail-area probability is readily interpreted as the fraction of 366 samples in which the simulated summary statistic was at least as large as the observed 367 value. As a consequence of the structure of the yield model, summary statistics that 368 measure the central tendency such as the mean and median are well represented by both 369 ensembles, so we focus on measures relevant to variability and the tails. 

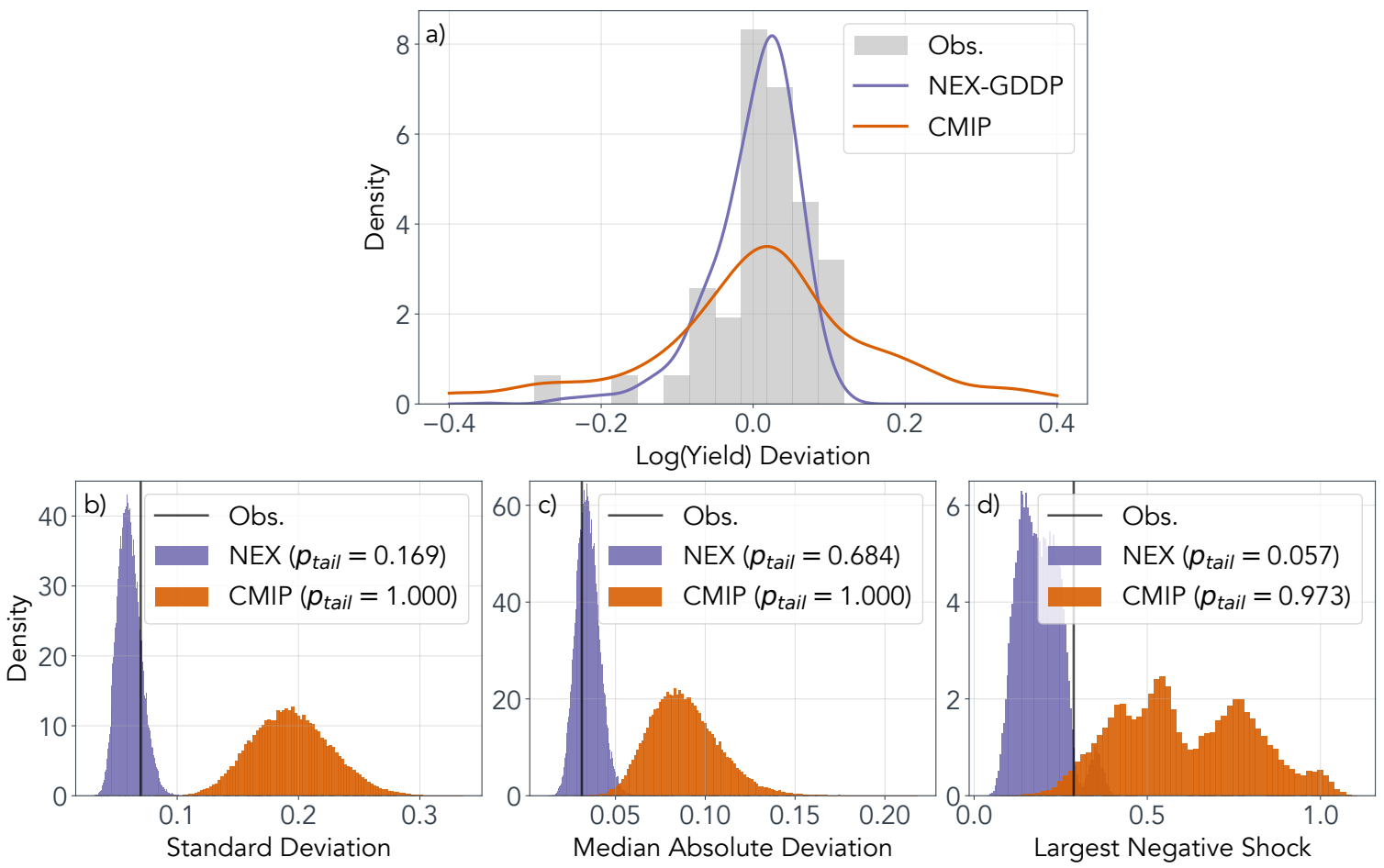

373

374 Figure 1. (top) National-level distribution of yield anomalies, modeled by each multi-model 375 ensemble: NEX-GDDP is shown in purple and CMIP in orange. The observational yield 376 distribution is shown as the gray histogram. (bottom) Sampling distribution of each 377 summary statistic: standard deviation (b), median absolute deviation (c), and magnitude 378 of largest negative yield shock (d). NEX-GDDP is shown in purple and CMIP in orange; 379 the single observed value is denoted by the black bar. Corresponding tail-area 380 probabilities are also stated. 


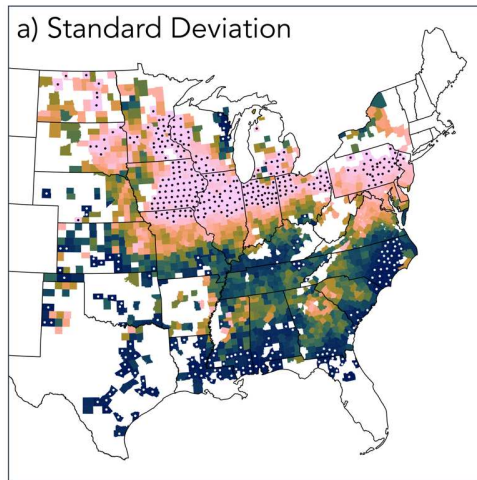

b) Median Absolute Deviation
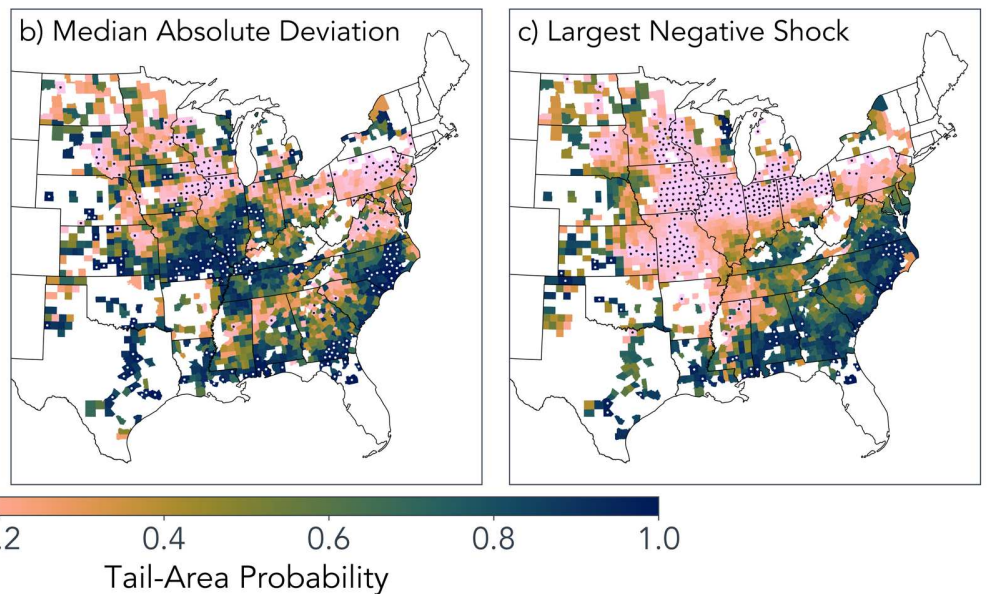

c) Largest Negative Shock

383 Figure 2. Tail-area probabilities of modeled maize yields for the NEX-GDDP ensemble

384 measured against observationally driven yields. Results are shown for standard deviation

385 (a), median absolute deviation (b), and the magnitude of the largest negative yield shock

386 (c). Stippling indicates tail-area probabilities less than 0.01 or greater than 0.99 . 

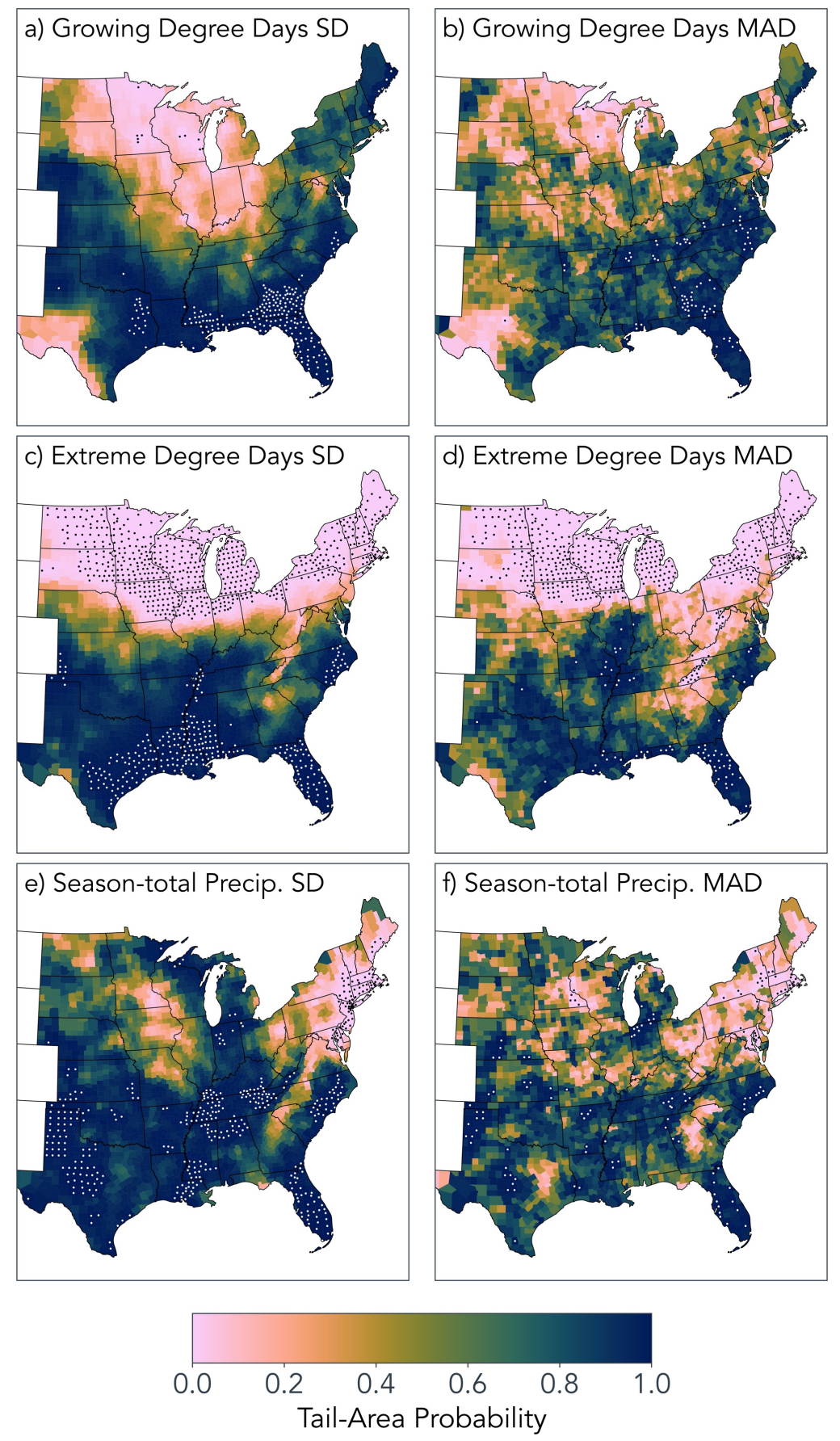

389 Figure 3. Tail-area probabilities for each climate variable used in the yield model, 390 calculated from the NEX-GDDP ensemble. The left column shows the results for standard 391 deviation; the right column for median absolute deviation (MAD). Climate variables are 392 organized by row: growing degree days (top), extreme degree days (middle), and 393 precipitation (bottom). Stippling indicates tail-area probabilities less than 0.01 or greater 394 than 0.99 . 

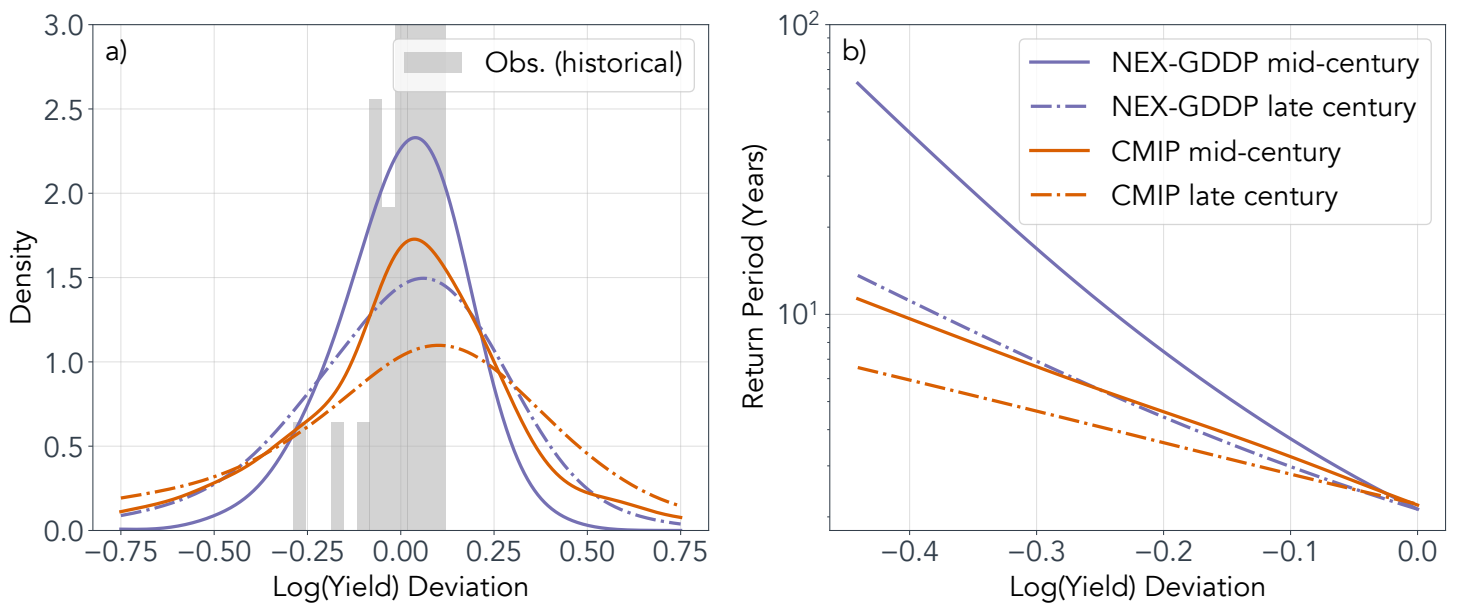

395

396

397 Figure 4. (a) RCP8.5 projections of U.S. yield variability for mid-century (2040-2059; full

398 lines) and late century (2080-2099; dashed lines). The NEX-GDDP projections are shown

399 in purple and the CMIP projections in orange; historical observations are also included for

400 reference as the gray histogram. (b) Corresponding return periods for increasingly large

401 magnitudes of negative yield shocks. In the absence of future yearly county-level

402 production shares, we aggregate to the national level by using the mean 1960-2016

403 weights. 


\section{References}

404 1. Change, Intergovernmental Panel on Climate. Climate Change 2014 -Impacts, 405 Adaptation and Vulnerability: Part A: Global and Sectoral Aspects: Working Group II

406 Contribution to the IPCC Fifth Assessment Report: Volume 1: Global and Sectoral

407 Aspects. vol. 1 (Cambridge University Press, 2014).

408 2. Huber, V. et al. Climate impact research: beyond patchwork. Earth Syst Dynam 5, 409 399-408 (2014).

410 3. Sriver, R. L., Forest, C. E. \& Keller, K. Effects of initial conditions uncertainty on 411 regional climate variability: An analysis using a low-resolution CESM ensemble.

412 Geophys Res Lett 42, 5468-5476 (2015).

413 4. Hawkins, E. \& Sutton, R. The Potential to Narrow Uncertainty in Regional Climate 414 Predictions. B Am Meteorol Soc 90, 1095-1107 (2009).

415 5. Hsiang, S. et al. Estimating economic damage from climate change in the United 416 States. Science 356, 1362-1369 (2017).

417 6. Ruijven, B. J. van, Cian, E. D. \& Wing, I. S. Amplification of future energy demand 418 growth due to climate change. Nat Commun 10, 2762 (2019).

419 7. Xu, C., Kohler, T. A., Lenton, T. M., Svenning, J.-C. \& Scheffer, M. Future of the 420 human climate niche. Proc National Acad Sci 117, 11350 (2020).

421 8. Cook, L. M., Anderson, C. J. \& Samaras, C. Framework for Incorporating Downscaled 422 Climate Output into Existing Engineering Methods: Application to Precipitation 423 Frequency Curves. J Infrastruct Syst 23, 04017027 (2017).

424 9. Anderson, J. et al. Progress on incorporating climate change into management of 425 California's water resources. Climatic Change 87, 91-108 (2008).

426 10. Martinich, J. \& Crimmins, A. Climate damages and adaptation potential across 427 diverse sectors of the United States. Nat Clim Change 9, 397-404 (2019).

428 11. Arnbjerg-Nielsen, K., Leonardsen, L. \& Madsen, H. Evaluating adaptation options 429 for urban flooding based on new high-end emission scenario regional climate model 430 simulations. Climate Res 64, 73-84 (2015).

431 12. Feser, F., Rockel, B., Storch, H. von, Winterfeldt, J. \& Zahn, M. Regional Climate 432 Models Add Value to Global Model Data: A Review and Selected Examples. B Am 433 Meteorol Soc 92, 1181-1192 (2011). 
434 13. Dixon, K. W. et al. Evaluating the stationarity assumption in statistically downscaled 435 climate projections: is past performance an indicator of future results? Climatic Change 436 135, 395-408 (2016).

437 14. Teutschbein, C. \& Seibert, J. Is bias correction of regional climate model (RCM) 438 simulations possible for non-stationary conditions? Hydrol Earth Syst Sc 17, 5061--5077 439 (2013).

440 15. Maraun, D. et al. Towards process-informed bias correction of climate change simulations. Nat Clim Change 7, 764-773 (2017).

442 16. Maraun, D. et al. Precipitation downscaling under climate change: Recent 443 developments to bridge the gap between dynamical models and the end user. Rev 444 Geophys 48, (2010).

445 17. Lanzante, J. R., Nath, M. J., Whitlock, C. E., Dixon, K. W. \& Adams-Smith, D. 446 Evaluation and improvement of tail behaviour in the cumulative distribution function 447 transform downscaling method. Int J Climatol 39, 2449-2460 (2019).

448 18. Lopez-Cantu, T., Prein, A. F. \& Samaras, C. Uncertainties in Future U.S. Extreme 449 Precipitation From Downscaled Climate Projections. Geophys Res Lett 47, (2020).

450 19. Maraun, D. Bias Correction, Quantile Mapping, and Downscaling: Revisiting the 451 Inflation Issue. J Climate 26, 2137-2143 (2013).

452 20. Walton, D. B., Sun, F., Hall, A. \& Capps, S. A Hybrid Dynamical-Statistical 453 Downscaling Technique. Part I: Development and Validation of the Technique. J Climate 454 28, 4597-4617 (2015).

455 21. Hertig, E. \& Jacobeit, J. A novel approach to statistical downscaling considering 456 nonstationarities: application to daily precipitation in the Mediterranean area. J Geophys 457 Res Atmospheres 118, 520-533 (2013).

458 22. Sippel, S. et al. A novel bias correction methodology for climate impact simulations. 459 Earth Syst Dynam 7, 71-88 (2016).

460 23. Cannon, A. J., Sobie, S. R. \& Murdock, T. Q. Bias Correction of GCM Precipitation 461 by Quantile Mapping: How Well Do Methods Preserve Changes in Quantiles and 462 Extremes? J Climate 28, 6938-6959 (2015).

463 24. Eum, H.-I., Cannon, A. J. \& Murdock, T. Q. Intercomparison of multiple statistical 464 downscaling methods: multi-criteria model selection for South Korea. Stoch Env Res Risk 465 A 31, 683-703 (2017). 
25. Teutschbein, C. \& Seibert, J. Bias correction of regional climate model simulations for hydrological climate-change impact studies: Review and evaluation of different methods. Journal of Hydrology 456-457, 12-29 (2012).

469 26. Glotter, M. et al. Evaluating the utility of dynamical downscaling in agricultural impacts projections. Proc National Acad Sci 111, 8776-8781 (2014).

27. Schlenker, W. \& Roberts, M. J. Nonlinear temperature effects indicate severe damages to U.S. crop yields under climate change. Proc National Acad Sci 106, 15594 (2009).

474 28. Schauberger, B. et al. Consistent negative response of US crops to high temperatures 475 in observations and crop models. Nat Commun 8, 13931 (2017).

476 29. Lobell, D. B., Bänziger, M., Magorokosho, C. \& Vivek, B. Nonlinear heat effects on 477 African maize as evidenced by historical yield trials. Nat Clim Change 1, $42-45$ (2011).

478 30. Lobell, D. B. et al. The critical role of extreme heat for maize production in the 479 United States. Nat Clim Change 3, 497-501 (2013).

480 31. D'Agostino, A. L. \& Schlenker, W. Recent weather fluctuations and agricultural 481 yields: implications for climate change. Agr Econ 47, 159-171 (2016).

482 32. Thrasher, B., Maurer, E. P., McKellar, C. \& Duffy, P. B. Technical Note: Bias 483 correcting climate model simulated daily temperature extremes with quantile mapping. 484 Hydrol Earth Syst Sc 16, 3309--3314 (2012).

485 33. Taylor, K. E., Stouffer, R. J. \& Meehl, G. A. An Overview of CMIP5 and the 486 Experiment Design. B Am Meteorol Soc 93, 485-498 (2012).

487 34. Bernard, L., Semmler, W., Keller, K. \& Nicholas, R. Improving Climate Projections 488 to Better Inform Climate Risk Management. (2015)

489 doi:10.1093/oxfordhb/9780199856978.013.0002.

490 35. Rigden, A. J., Mueller, N. D., Holbrook, N. M., Pillai, N. \& Huybers, P. Combined 491 influence of soil moisture and atmospheric evaporative demand is important for 492 accurately predicting US maize yields. Nat Food 1, 127-133 (2020).

493 36. Haqiqi, I., Grogan, D. S., Hertel, T. W. \& Schlenker, W. Quantifying the Impacts of 494 Compound Extremes on Agriculture and Irrigation Water Demand. Hydrology Earth Syst 495 Sci Discuss 2020, 1--52 (2020).

496 37. Knutti, R. Should we believe model predictions of future climate change?

497 Philosophical Transactions Royal Soc Math Phys Eng Sci 366, 4647-4664 (2008). 
38. Vuuren, D. P. van et al. The representative concentration pathways: an overview. Climatic Change 109, 5 (2011).

39. Herger, N. et al. Calibrating Climate Model Ensembles for Assessing Extremes in a

40. Sriver, R. L., Lempert, R. J., Wikman-Svahn, P. \& Keller, K. Characterizing uncertain sea-level rise projections to support investment decisions. Plos One 13, 504 e0190641 (2018).

505 41. Abatzoglou, J. T. \& Brown, T. J. A comparison of statistical downscaling methods suited for wildfire applications. Int J Climatol 32, 772-780 (2012).

507 42. Pierce, D. W., Cayan, D. R. \& Thrasher, B. L. Statistical Downscaling Using 508 Localized Constructed Analogs (LOCA)*. J Hydrometeorol 15, 2558-2585 (2014). 48, 1-33 (2020).

511 44. Abramowitz, G. et al. ESD Reviews: Model dependence in multi-model climate 512 ensembles: weighting, sub-selection and out-of-sample testing. Earth Syst Dynam 10, $91-$ 513105 (2019).

514 45. Moore, F. C., Baldos, U. L. C. \& Hertel, T. Economic impacts of climate change on 515 agriculture: a comparison of process-based and statistical yield models. Environ Res Lett $516 \mathbf{1 2}, 065008$ (2017).

517 46. Eiras-Barca, J. et al. Changes in South American hydroclimate under projected 518 Amazonian deforestation. Ann Ny Acad Sci 1472, 104-122 (2020).

519 47. DeAngelis, A. et al. Evidence of enhanced precipitation due to irrigation over the 520 Great Plains of the United States. J Geophys Res 115, (2010).

521 48. Sheffield, J., Goteti, G. \& Wood, E. F. Development of a 50-Year High-Resolution 522 Global Dataset of Meteorological Forcings for Land Surface Modeling. J Climate 19, 523 3088-3111 (2006).

524 49. Missirian, A. \& Schlenker, W. Asylum applications respond to temperature 525 fluctuations. Science 358, 1610-1614 (2017).

526 50. Ahmadalipour, A., Moradkhani, H. \& Svoboda, M. Centennial drought outlook over 527 the CONUS using NASA-NEX downscaled climate ensemble. Int J Climatol 37, 24775282491 (2017).

529 51. Thilakarathne, M. \& Sridhar, V. Characterization of future drought conditions in the 530 Lower Mekong River Basin. Weather and Climate Extremes 17, 47-58 (2017). 
531 52. Obradovich, N., Tingley, D. \& Rahwan, I. Effects of environmental stressors on daily 532 governance. Proc National Acad Sci 115, 8710--8715 (2018).

533 53. Diffenbaugh, N. S., Hertel, T. W., Scherer, M. \& Verma, M. Response of corn

534 markets to climate volatility under alternative energy futures. Nat Clim Change 2, 514$535518(2012)$.

536 54. Butler, E. E. \& Huybers, P. Adaptation of US maize to temperature variations. Nat 537 Clim Change 3, 68-72 (2013).

538 55. Conley, T. G. GMM estimation with cross sectional dependence. Journal of 539 Econometrics 92, 1-45 (1999). 


\section{Figures}
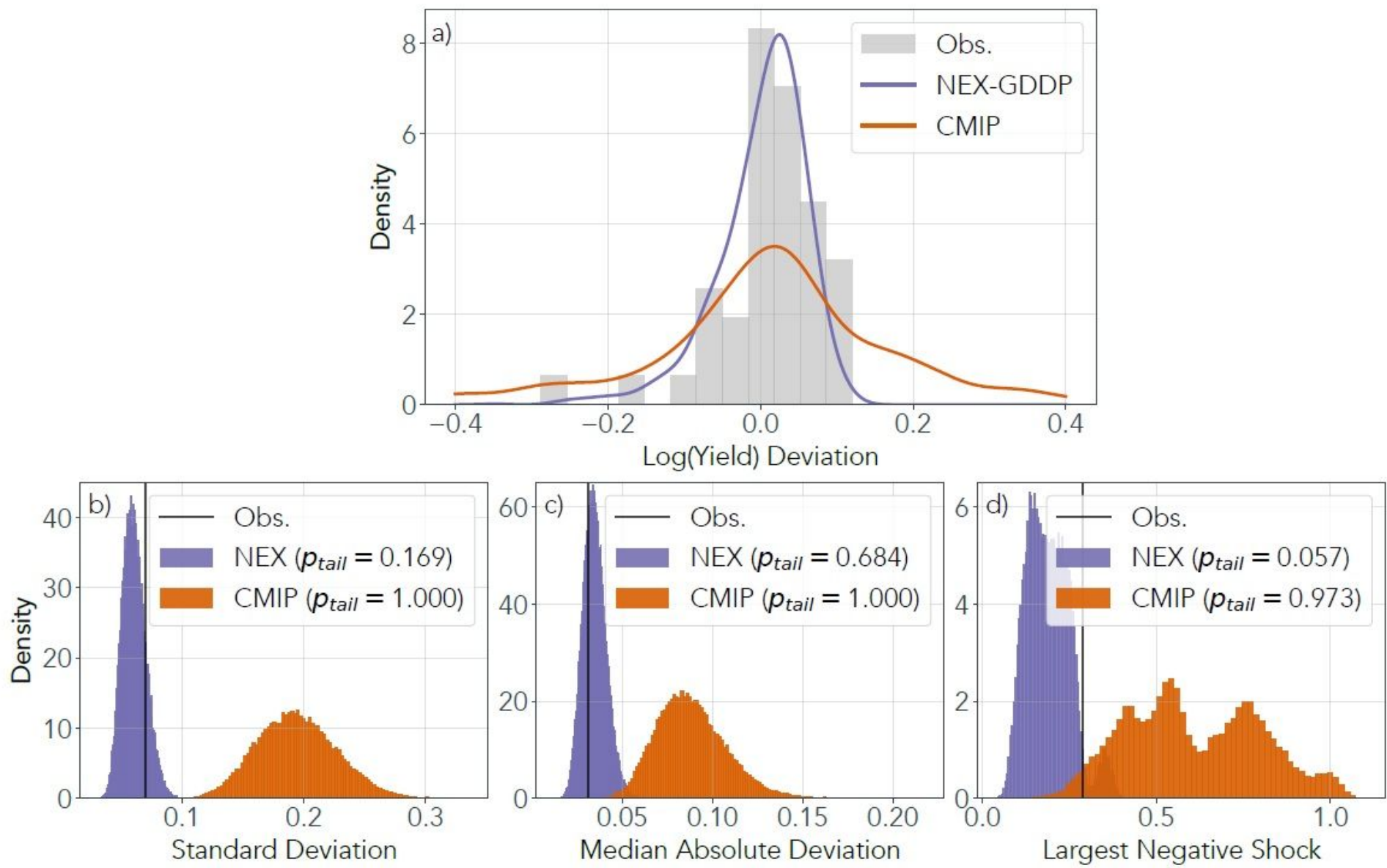

Figure 1

(top) National-level distribution of yield anomalies, modeled by each multi-model ensemble: NEX-GDDP is shown in purple and CMIP in orange. The observational yield distribution is shown as the gray histogram. (bottom) Sampling distribution of each summary statistic: standard deviation (b), median absolute deviation (c), and magnitude of largest negative yield shock (d). NEX-GDDP is shown in purple and CMIP in orange; the single observed value is denoted by the black bar. Corresponding tail-area probabilities are also stated. 


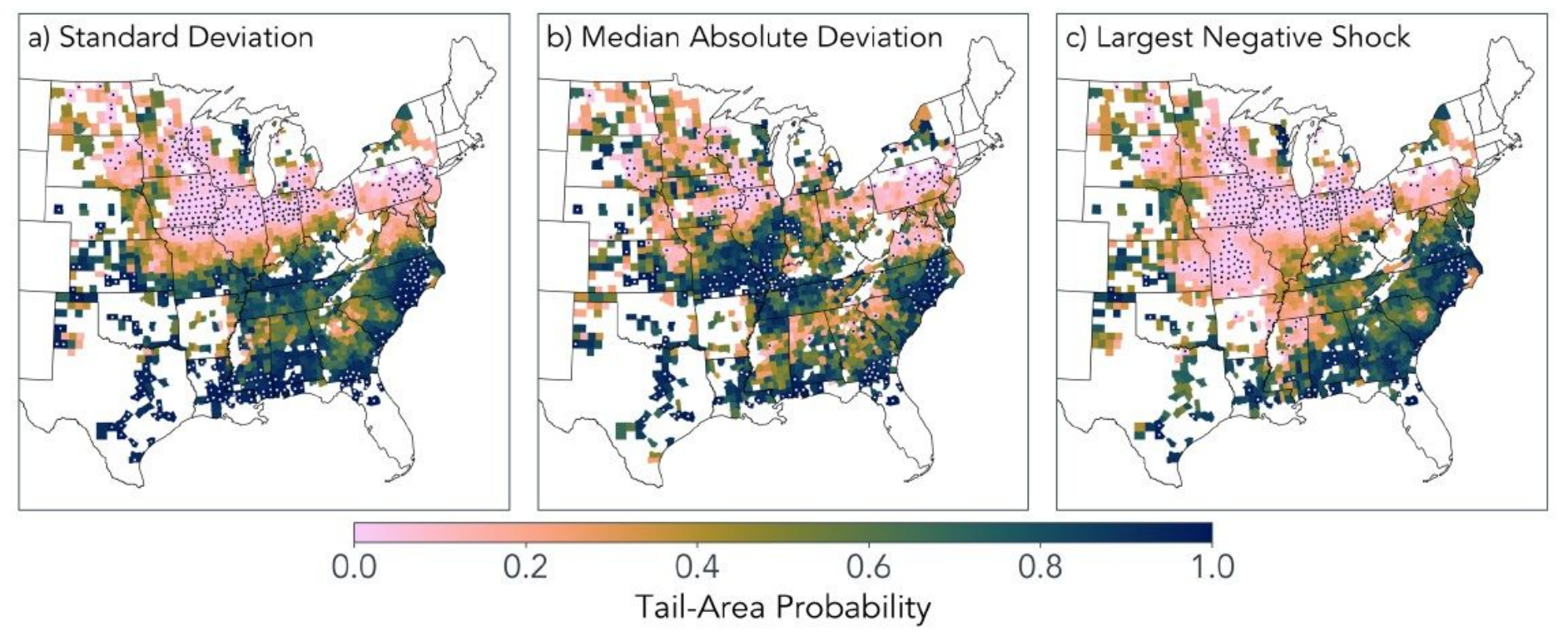

Figure 2

Tail-area probabilities of modeled maize yields for the NEX-GDDP ensemble measured against observationally driven yields. Results are shown for standard deviation (a), median absolute deviation (b), and the magnitude of the largest negative yield shock (c). Stippling indicates tail-area probabilities less than 0.01 or greater than 0.99 . 

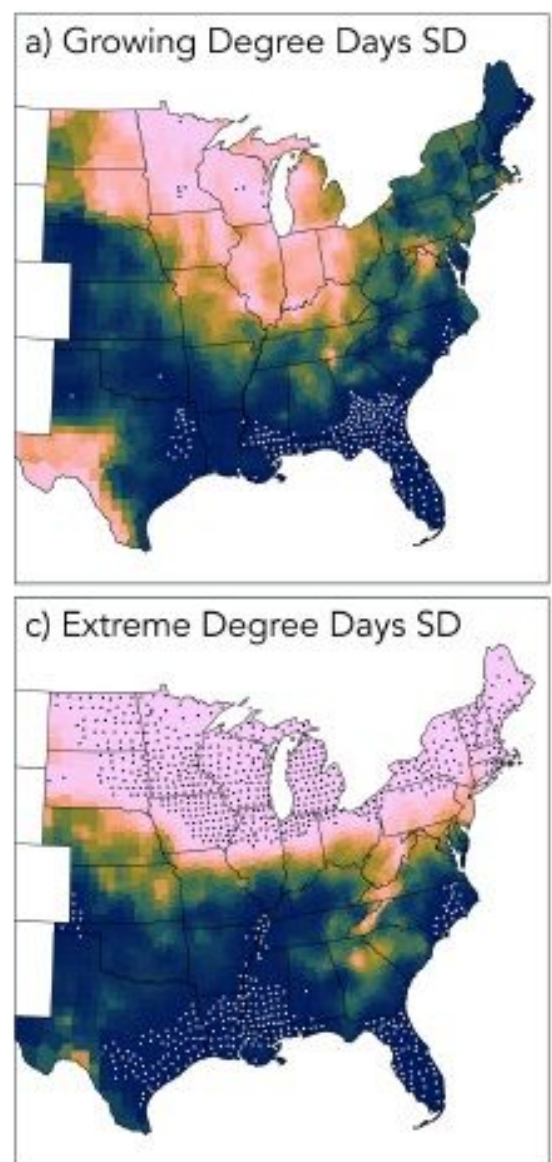

e) Season-total Precip. SD

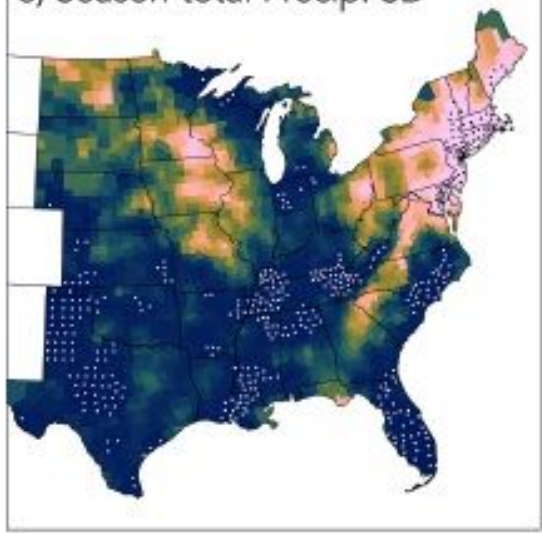

b) Growing Degree Days MAD

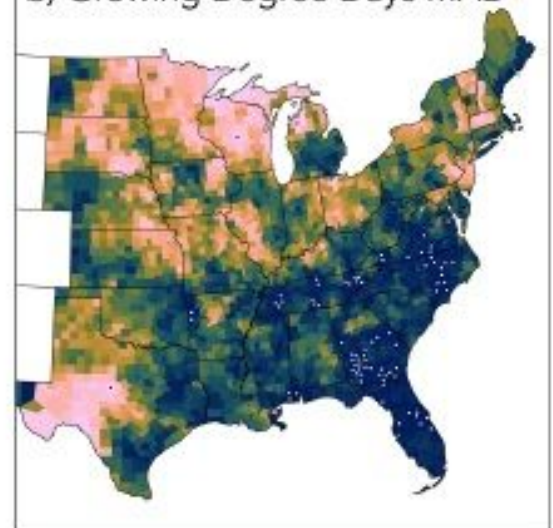

d) Extreme Degree Days MAD

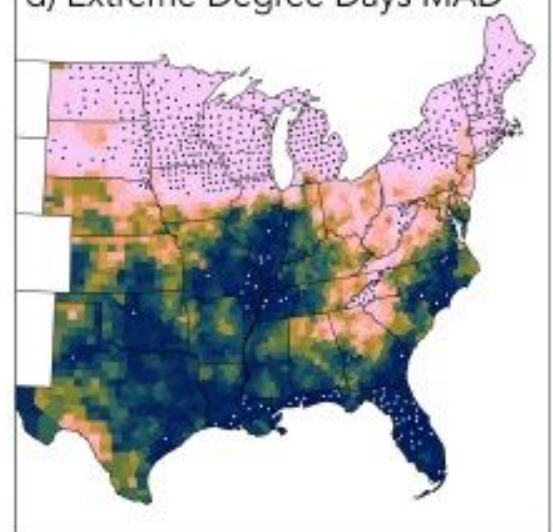

f) Season-total Precip. MAD

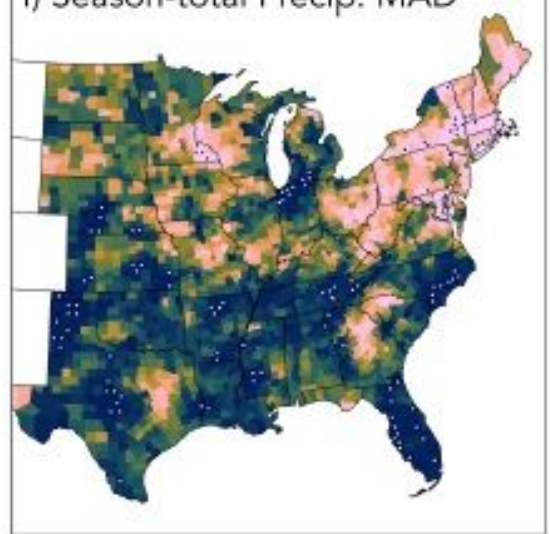

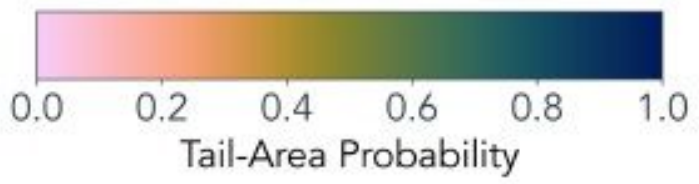

\section{Figure 3}

Tail-area probabilities for each climate variable used in the yield model, calculated from the NEX-GDDP ensemble. The left column shows the results for standard deviation; the right column for median absolute deviation (MAD). Climate variables are organized by row: growing degree days (top), extreme degree days (middle), and precipitation (bottom). Stippling indicates tail-area probabilities less than 0.01 or greater than 0.99 . 

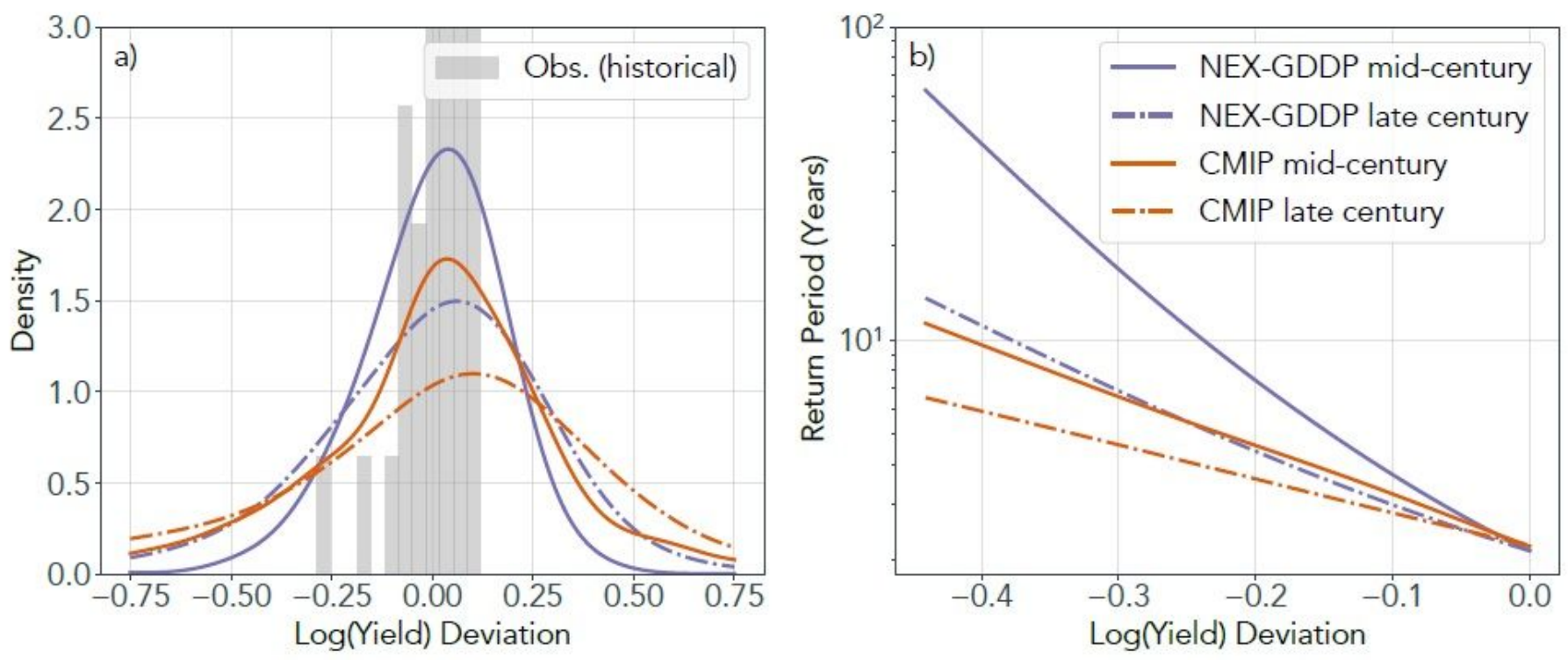

\section{Figure 4}

(a) RCP8.5 projections of U.S. yield variability for mid-century (2040-2059; full lines) and late century (2080-2099; dashed lines). The NEX-GDDP projections are shown in purple and the CMIP projections in orange; historical observations are also included for reference as the gray histogram. (b) Corresponding return periods for increasingly large magnitudes of negative yield shocks. In the absence of future yearly county-level production shares, we aggregate to the national level by using the mean 1960-2016 weights.

\section{Supplementary Files}

This is a list of supplementary files associated with this preprint. Click to download.

- NCCSIMaizeUncertaintyBCSD.pdf 